\title{
The pH-dependent photoluminescence of colloidal CdSe/ZnS quantum dots with different organic coatings $\dagger$
}

David Debruyne ${ }^{a, *}$, Olivier Deschaume ${ }^{a}$, Eduardo Coutiño-Gonzalez ${ }^{b}$, J.P. Locquet ${ }^{a}$, Johan Hofkens ${ }^{b}$, Margriet J. Van Bael ${ }^{a}$ and Carmen Bartic ${ }^{a, c}$

The photoluminescence of colloidal quantum dots (QDs) is known to be sensitive to the solution $\mathrm{pH}$. In this work we investigate the role played by the organic coating in determining the $\mathrm{pH}$-dependent photoluminescence (PL). We compare two types of CdSe/ZnS QDs equipped with different organic coatings, namely dihydrolipoic acid-capped QDs and phospholipid micelle-encapsulated QDs. Both QD types have their PL intensity quenched at acidic pH values, but they differ in terms of the reversibility of the quenching process. For dihydrolipoic acid-capped QDs, the quenching is nearly irreversible, with a small reversible component visible only on short time scales. For phospholipid micelleencapsulated QDs the quenching is notably almost fully reversible. We suggest that the surface passivation by the organic ligands is reversible for the micelle-encapsulated QDs. Additionally, both coatings display pH-dependent spectral shifts. These shifts can be explained by a combination of irreversible processes, such as photo-oxidation and acid etching, and reversible charging of the QD surface, leading to the quantum-confined Stark effect, the extent of each effect being coating-dependent. At high ionic strengths, the aggregation of QDs also leads to a spectral (red)shift, which is attributable to the quantum-confined Stark effect and/or electronic energy transfer.

\section{Introduction}

Quantum dots (QDs) or semiconductor nanocrystals display interesting photoluminescence (PL) properties including broad absorption and narrow emission in the UV/visible, good photostability and size-tunable emission color. Therefore QDs have been used as fluorescent probes in bio-imaging and labeling [1-3] and in biosensing [4,5]. Cadmium-based CdSe and CdTe QDs are among the best-studied colloidal QDs. The PL mechanism in CdSe-based QDs is governed by an exciton fine structure interacting with multiple trap states [6]. Trap states can originate from crystal surface atoms that are not fully coordinated. The passivation of surface traps greatly enhances the PL quantum yield. Surface passivation is achieved by the use of proper organic ligands and/or by overcoating the QD core with another semiconductor material such as ZnS, resulting in core/shell QDs $[7,8]$.

The synthesis of Cd-based QDs typically takes place in organic solvents [9]. The utilization of QDs in aqueous environments therefore requires a phase transfer step. Several methods exist to transfer QDs to the aqueous phase, including ligand exchange with thiols, the growth of a silica shell and encapsulation with amphiphilic copolymers or phospholipids [10]. Several reports have shown that the PL of water-soluble QDs is influenced by the pH. Most often, the $\mathrm{PL}$ intensity is quenched as the $\mathrm{pH}$ decreases [11-18]. However, thiol-stabilized CdTe QDs show the opposite trend $[19,20]$, and CdSe/ZnS QDs capped with poly(ethylene glycol)-appended dihydrolipoic acid (DHLA) were found to be pHinsensitive over the pH range $4-12$ [21]. The PL quenching under acidic conditions is reported to be irreversible [14,17], and is attributed to surface degradation mechanisms taking place in the presence of acid and oxygen [14]. 
Changes in the $\mathrm{pH}$ can also spectrally shift the PL, with the direction of the shift depending on the particular system. Mercaptopropionic acid-capped CdTe/ZnS QDs show a red shift with decreasing pH [17], while mercaptoacetic acidcapped CdSe/ZnS QDs, present a blue shift with decreasing pH [16], and streptavidin-functionalized poly(acrylic acid)coated CdSe/ZnS QDs lack a shift [14]. The origin of the pH-dependent spectral shift remains however unclear.

The previously mentioned studies show that the $\mathrm{pH}$-dependency of QD PL is variable and is affected by factors such as the semiconductor material ( $\mathrm{CdSe}, \mathrm{CdTe}, \mathrm{CdSe} / \mathrm{ZnS}$, etc.), the particle organic coating, and the history of the QDs. In the present paper, we investigate in detail the role of the organic coating by comparing the pH-dependent PL of DHLA-capped QDs and phospholipid-micelle encapsulated QDs. We examine the reversibility of the pH-dependency and propose a mechanism to explain the observed PL changes. We end with a discussion on the possible causes for the $\mathrm{pH}$-dependent spectral shift.

\section{Experimental}

\section{Materials}

CdSe/ZnS QDs (Lumidot ${ }^{\mathrm{TM}} \mathrm{CdSe} / \mathrm{ZnS} 640$ ) and ( \pm )- $\alpha$-Lipoic acid (synthetic, $\geq 99 \%$ ) were purchased from Sigma-Aldrich. 1,2distearoyl-sn-glycero-3-phosphoethanol-amine- $\mathrm{N}$-[carboxy(poly-ethylene glycol)-2000] (DSPE-PEG-COOH) and 1,2distearoyl-sn-glycero-3-phosphoethanolamine-N-[methoxy(polyethylene glycol)-2000] (DSPE-mPEG) were purchased from Avanti Polar Lipids. Rhodamine 6G (99\% Laser Grade) was purchased from Acros.

\section{Quantum dot water-stabilization}

(1) Purification step. CdSe/ZnS QDs in toluene were purified three times to remove excess ligands. Typically, $0.5 \mathrm{ml}$ of acetone/methanol mixture (1:1) was added to $0.5 \mathrm{ml}$ of the QD stock solution, upon which the color changed from bright red to turbid brown. The solution was centrifuged at $1000 \mathrm{~g}$ for $4 \mathrm{~min}$, causing precipitation of the QDs. The supernatant was discarded and the pellet redissolved in $0.5 \mathrm{ml}$ of toluene. This was repeated two more times, with the addition of chloroform instead of toluene after the final step.

(2) Phospholipid PEG ligand addition. 2.5 mg PEG phospholipids (DSPE-PEG-COOH/DSPE-mPEG in ratio 1:0 or 1:1) were dissolved in $0.5 \mathrm{ml}$ chloroform. The chloroform solution was added to the purified QDs and the solution was shaken for at least 2 hours, after which the chloroform was evaporated under streaming nitrogen. The dried QDs were redispersed in $0.5 \mathrm{ml}$ of ultrapure water.

(3) Dihydrolipoic acid ligand exchange. $3.75 \mathrm{mg}$ of lipoic acid and $2.5 \mathrm{mg}$ of NaBH4 were each dissolved separately in 1.25 $\mathrm{ml}$ borate buffer $(250 \mathrm{mM}, \mathrm{pH} 9.0)$ at $0^{\circ} \mathrm{C}$. Both solutions were then mixed and allowed to react for two hours, allowing the lipoic acid to be reduced to dihydrolipoic acid (DHLA). Next, $0.5 \mathrm{ml}$ of purified QDs in chloroform was diluted to $2.5 \mathrm{ml}$ and the DHLA solution was added. The biphasic mixture was vortexed for 15 minutes, resulting in the transfer of QDs from the chloroform to the water phase. The water phase was collected and purified by dialysis against borate buffer ( 2.5 $\mathrm{mM}, \mathrm{pH}$ 9.0).

\section{Sample preparation}

For measurements of the steady-state PL, a 96-well plate was used as a sample container. A Britton-Robinson buffer was used to achieve a wide range of $\mathrm{pH}$ values. This buffer is prepared by mixing equimolar amounts of acetic, phosphoric, and boric acid, and adjusting the $\mathrm{pH}$ with sodium hydroxide. Each well of the plate contained the buffer solution at a 
A

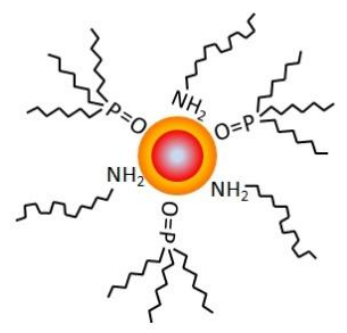
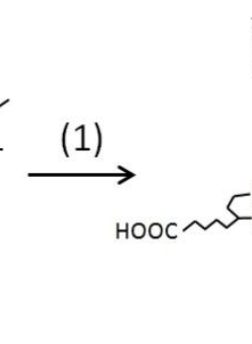

(2)

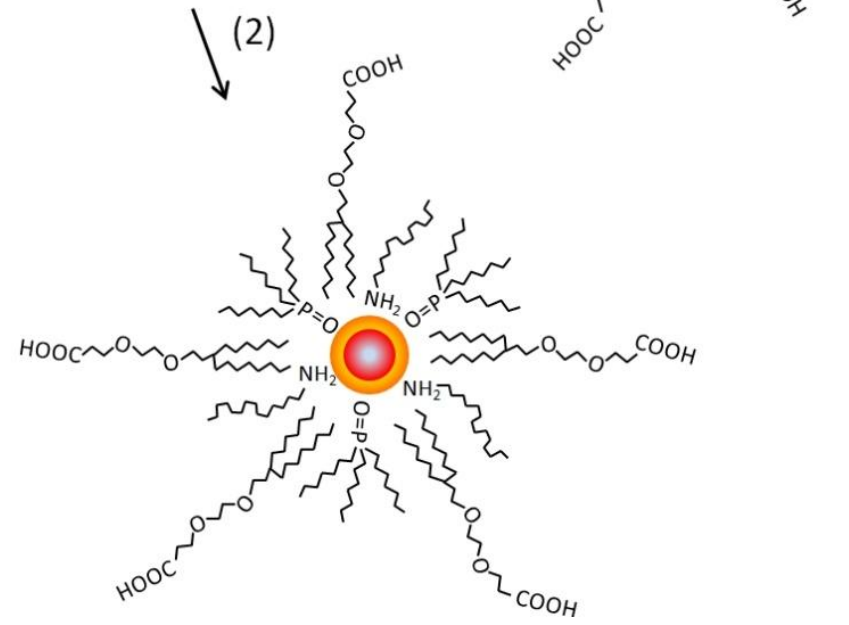

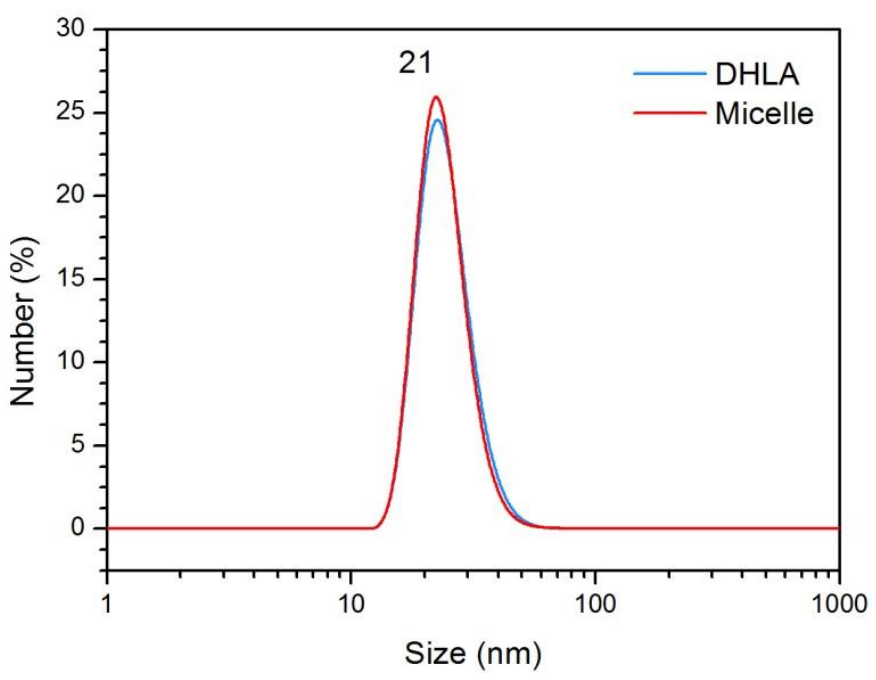

Figure 1. (A) Schematic representation of the water-solubilization methods used in this work: (1) ligand exchange with dihydrolipoic acid (DHLA), and (2) ligand addition with PEG-phospholipids (micellization). (B) Number distributions of the particle hydrodynamic size at neutral pH obtained from dynamic light scattering measurements.

specific $\mathrm{pH}$. Aliquots of the QD solution were added as the final step of sample preparation. The reversibility measurements of (Figure 4 and Figure 5) were performed in PS fluorescence cuvettes. To buffer the solution we used a mixture of citric and boric acid. During the experiment, the $\mathrm{pH}$ value was adjusted by adding hydrogen chloride or sodium hydroxide.

\section{Measurements and data analysis}

Measurements of the pH were done with a S220 pH Seven Compact pH meter from Mettler Toledo. PL spectra and intensities were obtained with an Infinite M200 Pro plate reader. For the reversibility experiments, PL spectra were acquired with a QuantaMaster ${ }^{\mathrm{TM}} 60$ from Photon Technology International. QDs were excited at $500 \mathrm{~nm}$. All PL spectra were baseline-corrected. For the reversibility measurements, the PL intensities were corrected for the (small) dilution effect caused by the addition of acid/base. The average PL emission wavelength shown in figures is the intensity-weighted average wavelength. When the intensity of the PL spectrum was too low to reliably determine the average, no value is shown. UV/visible absorption spectra were acquired with an Ultrospec 2100 pro spectrophotometer from GE Healthcare. The hydrodynamic size and zeta potential distributions were measured with a Malvern Zetasizer Nano ZS. Values for the absolute external quantum yield (QY) was obtained using an integrating sphere coupled to a Horiba Jobin Yvon fluorolog FL3-22 fluorimeter [22].

\section{Results and discussion}




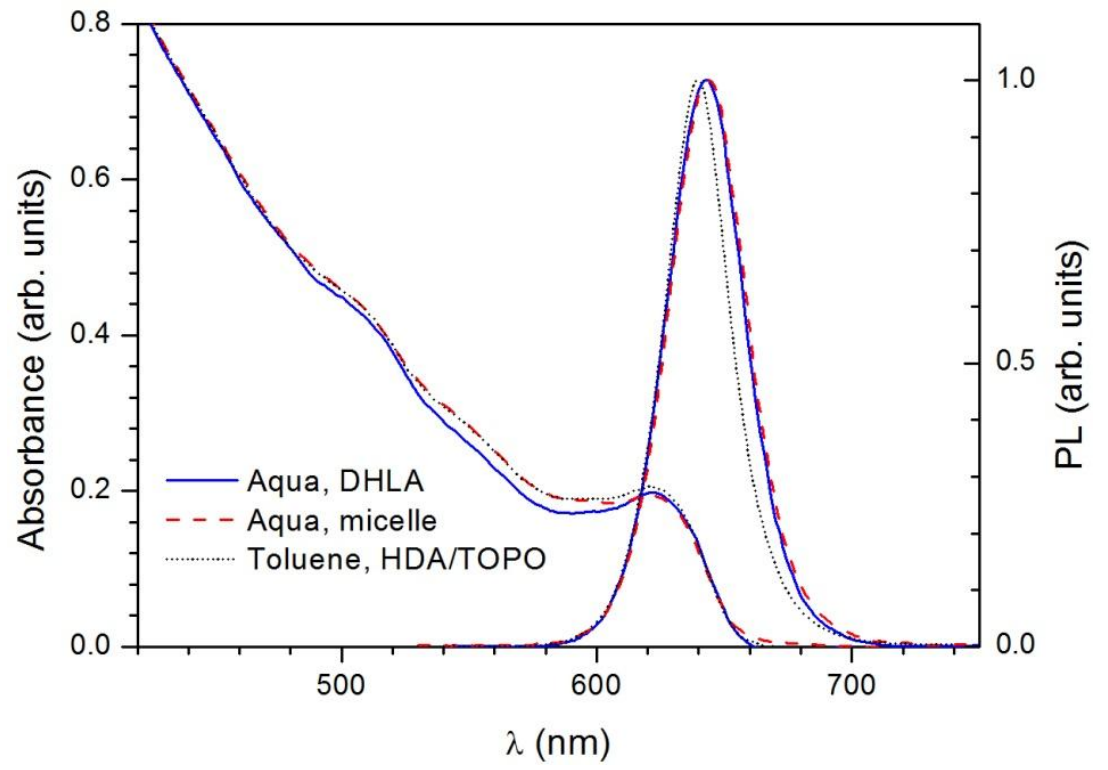

Figure 2. Absorption and (normalized) PL spectra of CdSe/ZnS QDs in different media.

\section{Water-solubilization and basic PL properties}

Commercially available CdSe/ZnS QDs dispersed in toluene were transferred to water by either ligand exchange or ligand addition (Figure 1A). The ligand exchange method replaces the original hexadecylamine (HDA) and trioctylphosphine oxide (TOPO) ligands by dihydrolipoic acid (DHLA). This dithiol coordinates strongly to the nanocrystal surface by cooperatively binding its two thiol anchoring groups [23]. The ligand addition method on the other hand, preserves the original ligands by encapsulating the lipophilic particles inside phospholipid-PEG micelles. Both water solubilization procedures increase the particle size substantially. The inorganic core size determined by TEM is $5.7 \pm 0.5 \mathrm{~nm}$ (SI figure 1). For the water-soluble QDs, the hydrodynamic diameter as measured by dynamic light scattering is approximately $21 \mathrm{~nm}$ for both coatings (Figure 1B). Both coatings have a negative $\zeta$-potential due to the presence of carboxylic acid groups in the organic ligands. At neutral $\mathrm{pH}$, the $\zeta$-potential is $-11 \pm 6 \mathrm{~nm}$ for micelle-encapsulated QDs and $-14 \pm 14 \mathrm{~nm}$ for DHLAcapped QDs.

The absorption spectrum appears unchanged after phase transfer, whereas the PL spectrum is slightly red-shifted (Figure 2). The small redshift may be a manifestation of the quantum-confined Stark effect (QCSE), caused by a non-uniform coverage of organic ligands after phase transfer. The non-uniform coverage can create an internal electric field that polarizes the exciton and lowers its energy [24]. A common issue is the decrease in PL quantum yield (QY) after phase transfer [25-28]. The QY is 34\% when the QDs are dispersed in toluene, but after transfer to water, the QY is typically smaller than $10 \%$ for micelle-encapsulated QDs, and smaller than $1 \%$ for DHLA-capped QDs (the exact QY value depends on the $\mathrm{pH}$, storage time, and experimental batch). A decrease in the PL QY is usually due to the appearance of surface traps that originate from interfacial crystal atoms that are not fully coordinated [25]. These surface traps enable nonradiative recombination of the exciton and thereby decrease the QY. Additionally, the PL QY could be influenced by the QCSE, which is known to decrease the PL intensity [29]. The surface ligand coverage is typically incomplete, and those surface atoms that are not passivated may be slightly charged [6], causing an internal electric field. 
A

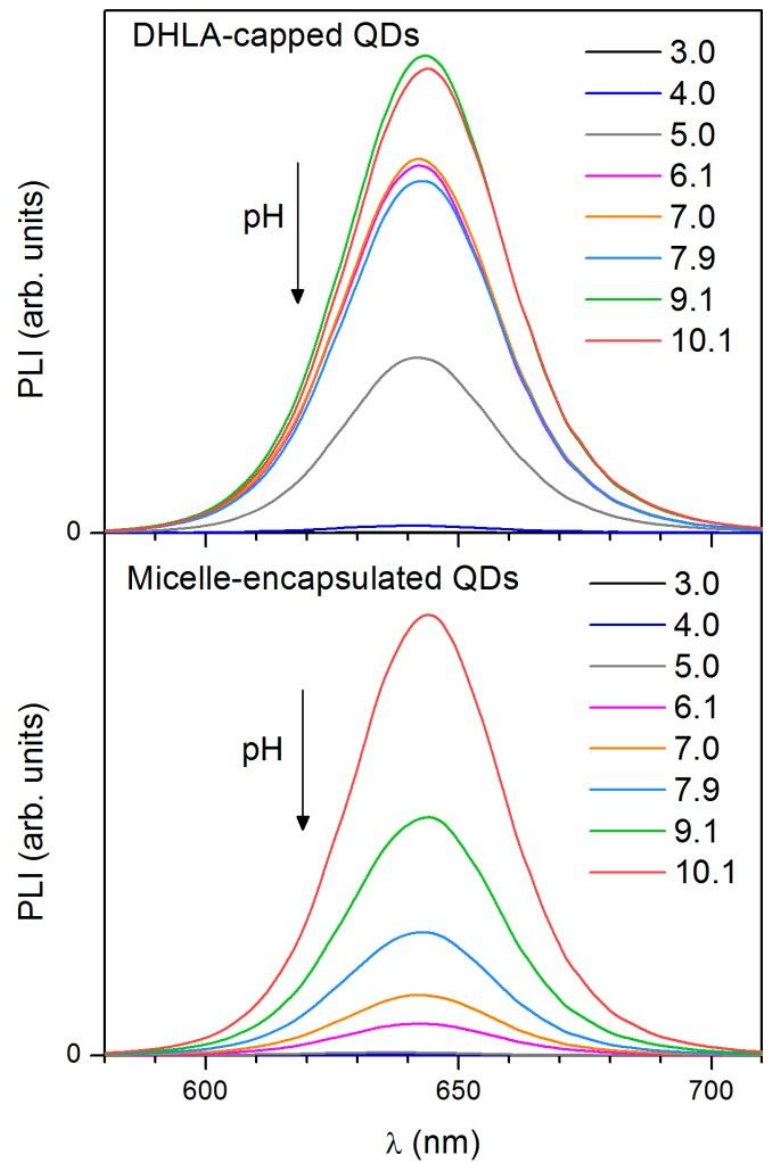

B

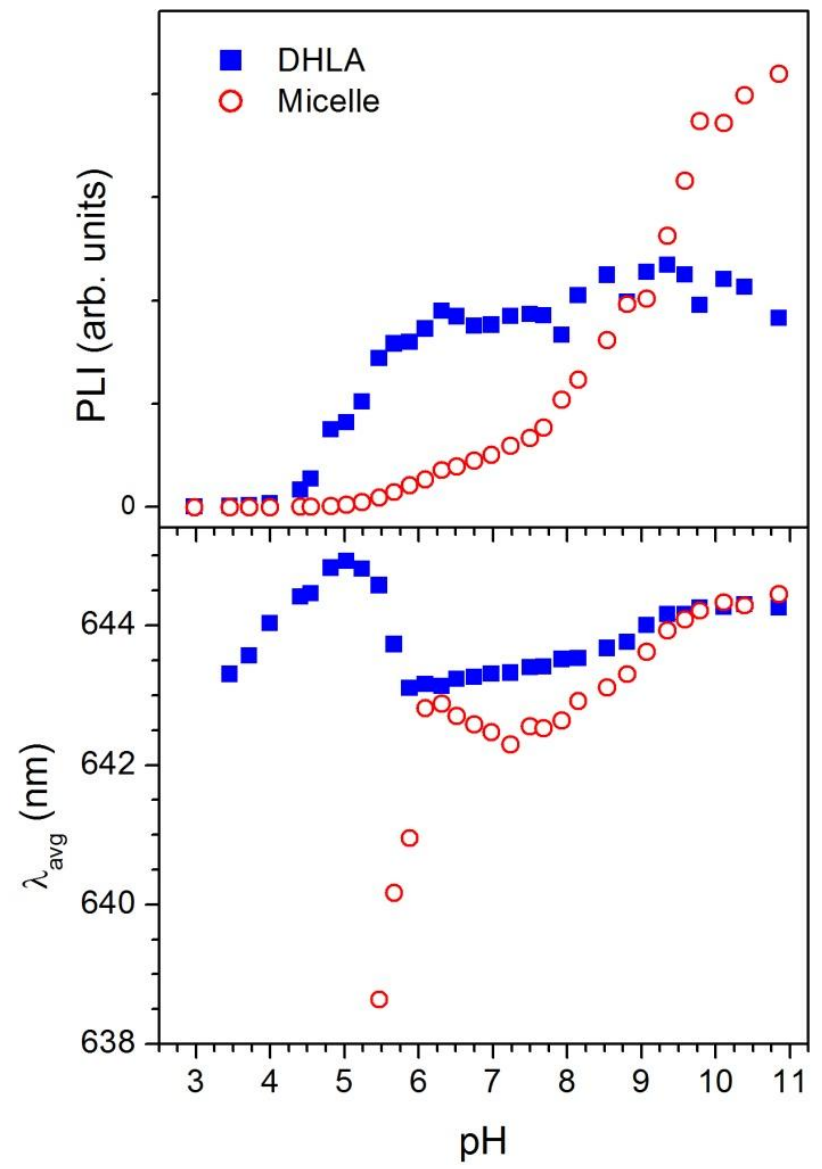

Figure 3. (A) pH-dependence of PL spectra for DHLA-capped and micelle-encapsulated QDs. (B) Integrated intensities and average PL wavelength as a function of the $\mathrm{pH}$. Each $\mathrm{pH}$ value represents an individual sample. The PL intensities of DHLA-capped and micelleencapsulated QDs are scaled to fit on the same graph.

Surface atoms can be passivated by organic ligands, typically HDA and TOP(O) in the organic solvent phase. HDA forms dative covalent bonds with $\mathrm{Zn}$ atoms at the nanocrystal surface by sharing its nitrogen free electron pair [30,31]. HDA thus passivates hole traps originating from $\mathrm{Zn}$ atoms with empty orbitals. Previous studies have demonstrated that part of the ligands are reversibly bound to the crystal surface. Dilution of the sample solution can therefore cause dissociation of these ligands, which leads to a reduction in the PL QY [32]. The purification step performed at the beginning of the watersolubilization process (see experimental section) may therefore lead to ligand dissociation and a reduction of the QY. The sample is indeed visibly less bright after purification. In the ligand addition method, these ligand-deficient QDs are then encapsulated inside micelles, which explains why micelle-encapsulated QDs have a lower QY than the original lipophilic QDs. In the ligand exchange method, the dissociation of ligands is irrelevant, as the original ligands are replaced with DHLA. In this case however, the ligand exchange itself is deleterious to the QY. Thiol-ligand exchange is indeed known to reduce the PL intensity [16].

\section{The pH-dependence of the photoluminescence intensity}

Figure $3 \mathrm{~A}$ shows the $\mathrm{PL}$ spectra of QDs as a function of the $\mathrm{pH}$. For both coating types, the $\mathrm{PL}$ is quenched at low $\mathrm{pH}$ values, in accordance with typical observations [14-17]. Measurements of the PL decay for micelle-encapsulated QDs show that the PL quenching proceeds along with a decrease of the PL lifetime (fig. S3). There is thus an increase in the 
non-radiative recombination rate at low $\mathrm{pH}$, meaning that the PL quenching is caused by a decrease in the PL QY. Closer inspection reveals that the two coatings show a different behavior in terms of the onset of PL quenching and the sensitive $\mathrm{pH}$ range (Figure 3B). For micelle-encapsulated QDs, the PL intensity increases steeply between $\mathrm{pH} 6$ and $\mathrm{pH} 10$. For DHLA-capped QDs, the PL intensity is fairly constant above pH 6, but it rapidly declines below pH 6.

The different $\mathrm{pH}$-dependence can be explained by differences in the organic passivation layer. The $\mathrm{pH}$ affects the protonation state of the organic ligands and therefore their ability to passivate the surface. As discussed in the previous section, non-passivated surface sites can cause a decrease in the PL QY. For micelle-encapsulated QDs, the surface ligands are HDA and TOP(O). HDA has a pKa value of 10.6 [33], which means that the protonated state $\left(\mathrm{RNH}_{3}{ }^{+}\right)$dominates below $\mathrm{pH}$ 10.6. However, it is the deprotonated state $\left(\mathrm{RNH}_{2}\right)$ that is responsible for passivating hole traps [30]. Therefore, a decrease in PL intensity can be expected below pH 10.6 for micelle-encapsulated QDs, which is indeed observed in Figure 3B. DHLA has a pKa value similar to HDA, namely 10.7 [34]. For thiols however, the deprotonated form (RS-) is not beneficial for the PL QY. Thiolates (RS-) presumably passivate electron traps by their electron-donating ability, but they introduce new hole traps at high concentrations [35]. The measured pH range lies mostly below the pKa of DHLA, where the protonated form (RSH) is dominant. Therefore, we expect no decrease of the PL intensity below pH 10.7. This is confirmed in Figure 3 by the almost constant PL intensity between pH 6 and 10. Below pH 6 however, a quick decline of the PL intensity can be observed. At acidic pH, QDs are unstable against acid etching [25]. Durisic and co-workers have suggested that the ZnS shell of CdSe/ZnS QDs slowly decomposes in the presence of acid and oxygen [14]. The process is supposedly self-limited by the formation of a sulfur-rich layer, with the excess sulfur atoms acting as hole traps, thereby diminishing the PL intensity.

\section{Reversibility of the pH-dependent PL intensity changes}

Additional information on the $\mathrm{pH}$ mechanism can be obtained by investigating the reversibility of the quenching process. Irreversible PL quenching due to acidic $\mathrm{pH}$ has been reported for 3-thiopropionic acid-capped QDs [17] and for QDs coated with poly(acrylic acid) (PAA) [14]. The irreversible character stems from the permanent damage done to the crystal (surface) at low pH [14]. There are few reports on reversible pH quenching. In one work, reversibility was demonstrated for QDs confined in a polyelectrolyte matrix covered with a pH-responsive hydrogel of poly(methyl methacrylate) [19].

We tested the reversibility of the $\mathrm{pH}$ quenching by cycling between $\mathrm{pH}$ values 9 and 5 (Figure 4). Upon exposure to $\mathrm{pH} 5$, the PL intensity of both QD types is strongly quenched as expected. When the $\mathrm{pH}$ is restored to 9 , the $\mathrm{PL}$ intensity of micelle-encapsulated QDs recovers almost completely, whereas the PL intensity of DHLA-capped QDs remains quenched. Notice that the change in PL intensity at the transition from pH 9 to $\mathrm{pH} 5$ is more gradual for micelle-encapsulated QDs than for DHLA-capped QDs. Figure 5 shows the same experiment, but with a shorter time scale and with more $\mathrm{pH}$ cycles. For both QD types, each increase/decrease of the $\mathrm{pH}$ is associated with an increase/decrease of the $\mathrm{PL}$ intensity. Interestingly, also DHLA-capped QDs display some reversibility on this time scale, although overall the PL intensity decreases with each pH cycle. 


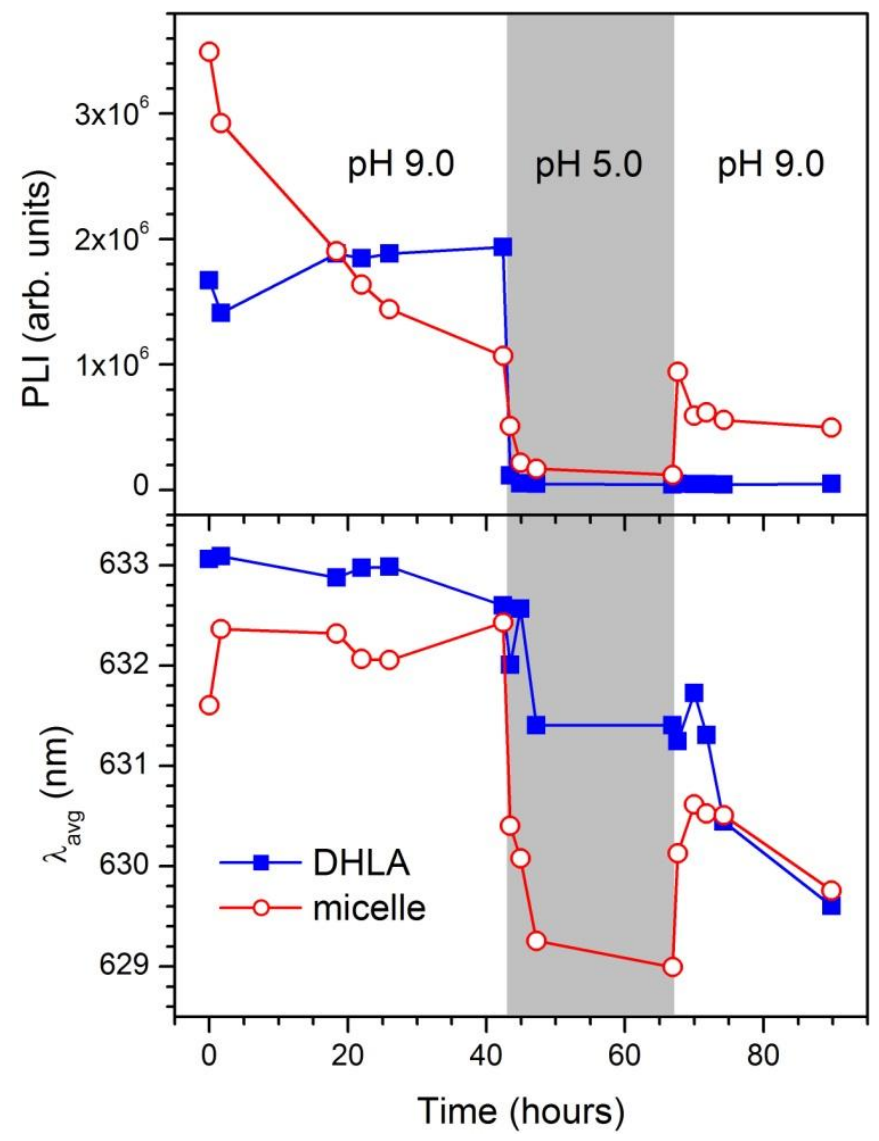

Figure 4. Test of the reversibility of the PL quenching at low pH. The PL intensity (PLI) and average PL wavelength ( $\lambda_{\text {avg }}$ ) are plotted versus time while the sample $\mathrm{pH}$ is changed from 9 to 5, and back to 9. The PLI was first allowed to stabilize at pH 9, however the stabilization for micelle-encapsulated was still ongoing after $\mathbf{4 0} \mathrm{hrs}$.

The PL intensity quenching is thus mainly reversible for micelle-encapsulated QDs. For DHLA-capped QDs, the PL intensity quenching is irreversible on the long time scale, but the shorter time scale reveals also a small reversible component. The reason for the different behavior should again be sought in the different organics surrounding the two particle types. DHLA ligands form a thin, possibly incomplete, monolayer around the QDs. Consequently, the QD surface is easily accessible to reactive species in the solution and irreversible damage by photo-oxidation or acid etching can quickly proceed at low pH. Micelle-encapsulated QDs, on the other hand, are better protected by a hydrophobic bilayer that surrounds the particles. The bilayer may serve as a barrier to the diffusion of oxygen and other hazardous species that would damage the QD surface [25]. In the previous section, we proposed that the PL intensity quenching for micelleencapsulated QDs is mainly a consequence of surface depassivation. At low pH, part of the HDA ligands is protonated and therefore unavailable for surface passivation. The protonated ligands stay inside the micelle because they are insoluble in water. Once the $\mathrm{pH}$ is restored to higher values, the ligands are again deprotonated and they can repassivate the surface, explaining the reversible $\mathrm{pH}$ quenching in the case of micelle-encapsulated QDs.

\section{pH-dependent spectral shift}

Modifying the sample pH also causes spectral emission shifts of several nanometers (Figure 3). The origin of these shifts is less clear, as they are more subtle than the PL intensity changes. The spectral shifts usually occur simultaneously with 


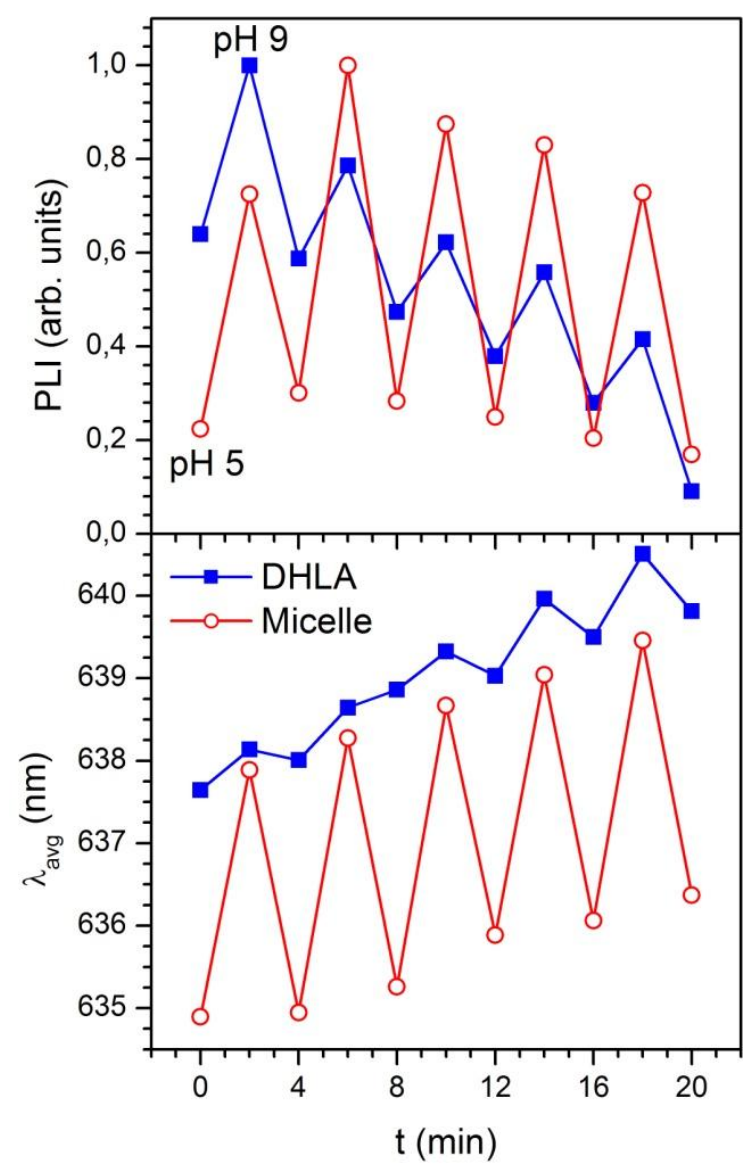

Figure 5. Test of the reversibility with quick pH cycling every two minutes. The PL intensity (PLI) and average PL wavelength ( $\left.\lambda_{\text {avg }}\right)$ are plotted versus time while the $\mathrm{pH}$ is cycled between 5 and 9.

large changes in the PL intensity. For instance, the decrease in PL intensity of DHLA-capped QDs below pH 6 is associated with a pronounced redshift $(\sim 2 \mathrm{~nm}$ ) between $\mathrm{pH} 5$ and 6 (Figure 3).

Figure 4 shows that the PL intensity quenching upon exposure to $\mathrm{pH} 5$ is accompanied by a spectral blueshift of $\sim 1 \mathrm{~nm}$ for DHLA-capped QDs and $\sim 3 \mathrm{~nm}$ for micelle-encapsulated QDs. When the $\mathrm{pH}$ is restored to 9 , the average PL wavelength is red-shifted for micelle-encapsulated QDs, but for DHLA-capped QDs there is no clear redshift. Interestingly, after the pH has been restored to 9, the average PL wavelength starts to blue-shift over time. In Figure 5, the exposure time to pH 5 is limited by the quicker cycling of the $\mathrm{pH}$. In this case, the spectral shift is almost fully reversible for micelle-encapsulated QDs and also the DHLA-capped QDs show a reversible component (albeit small and against a rising background). Figure 4 and Figure 5 show that there is both a reversible and irreversible component to the spectral shifts.

Mainly two mechanisms causing spectral shifts have been reported in literature. Firstly, etching of the nanocrystal by photo-oxidation or acids causes a blueshift of the PL and the absorption spectrum, because the size of the nanocrystal is reduced $[25,36]$. This spectral blueshift is permanent and irreversible. Secondly, the quantum-confined Stark effect (QCSE), which is due to polarization of the exciton state by an electric field, causes a spectral redshift $[37,38]$. The electric field causing the QCSE can be externally applied or result from local surface charges originating from unpassivated surface states [39]. It was shown that fluctuating surface charges cause spontaneous spectral diffusion in single QDs [40,41]. Spectral shifting due to the QCSE is reversed when the electric field is removed. Braam and co-workers recently explained 


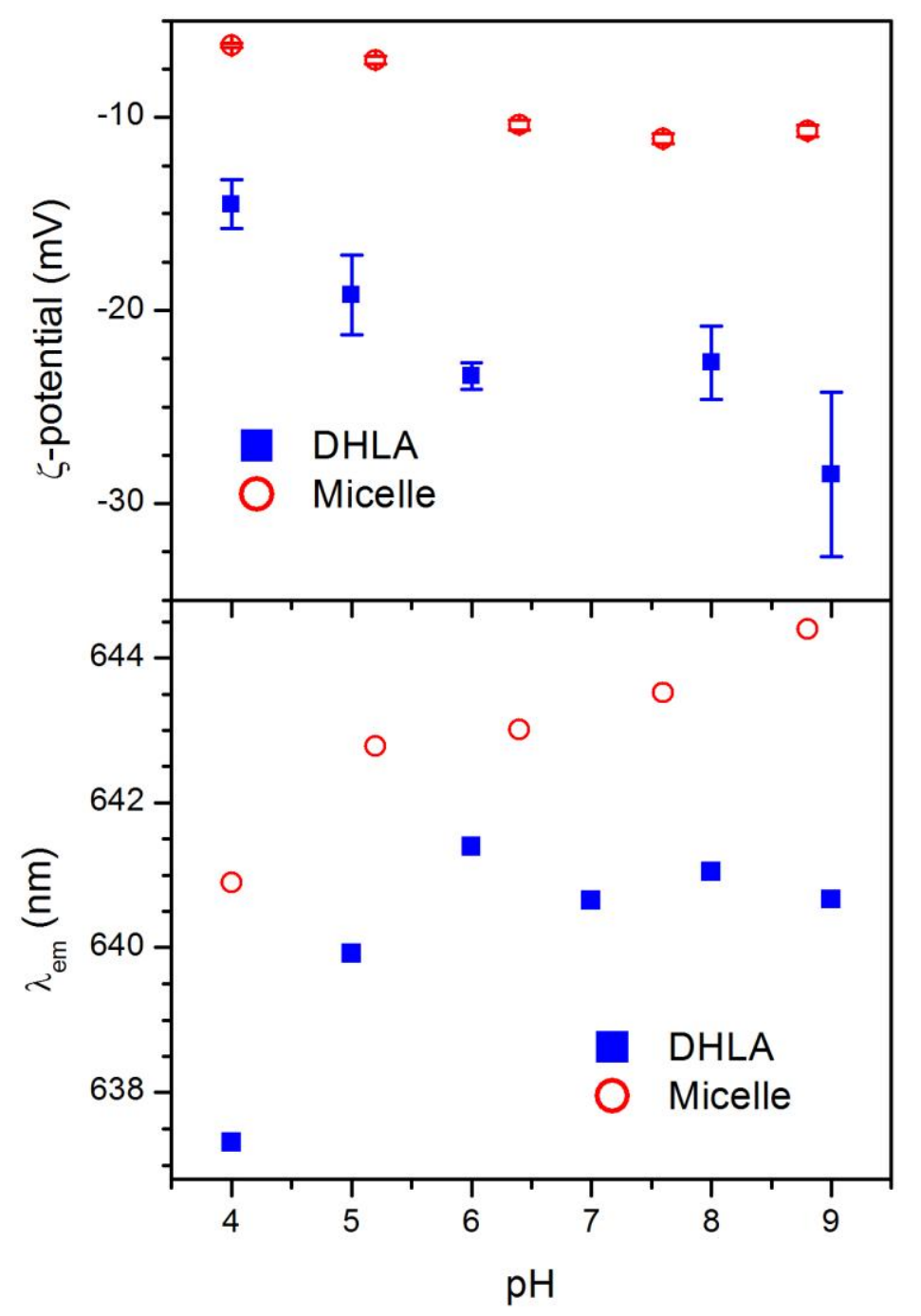

Figure 6. Effect of the zeta potential on the PL average wavelength.

the spectral diffusion of single CdSe/ZnS QDs embedded in poly(methyl methacrylate) by a combination of the above two mechanisms [41].

In our case, the spectral shifting possesses both a reversible and an irreversible component. Therefore we can, like Braam and co-workers, expect a combination of acid or photo-oxidative etching and the QCSE. The presence of irreversible etching is evident at low $\mathrm{pH}$ values $(\leq 5)$. In this $\mathrm{pH}$ regime, we were unable to measure the typical absorption spectrum of QDs after some days, most likely because the QDs were completely etched and dissolved. At milder pH values (above 5), the absorption spectrum appears normal and is not blue-shifted. According to Durisic and co-workers, etching can still occur in this $\mathrm{pH}$ regime, but it proceeds more slowly and is self-limited by the formation of a sulfur-rich layer [14]. We expect that for the core/shell QDs used in this work, only the shell is affected and the CdSe core is left intact. This would explain the absence of a blueshift in the absorption spectrum.

The reversible component can be explained by the QCSE, but only if the charging process that causes the electric field is reversible itself. The magnitude of the spectral shifts (a few $\mathrm{nm}$ ) is in accordance with typical values observed for the QCSE [24,39-41]. Because our system (consisting of QDs, organic ligands, and buffer ions) is highly complex, it is difficult to identify the exact charge source(s) that are responsible for the QCSE. Below we list some (surface) charge sources that 


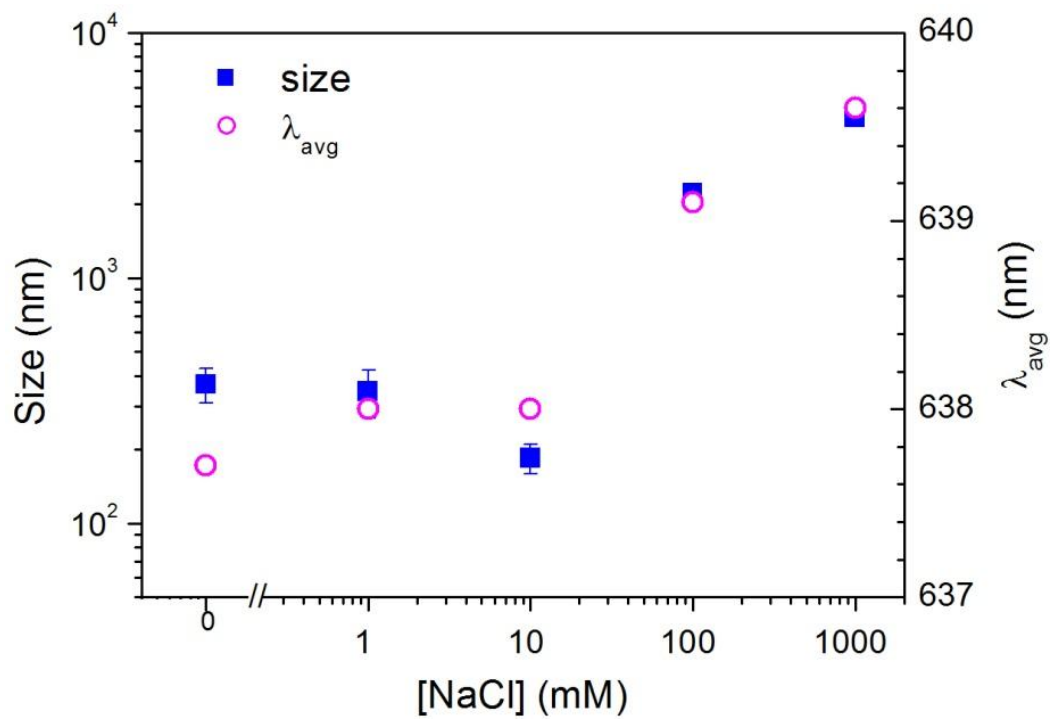

Figure 7. Effect of ionic strength on the average hydrodynamic size (Z-avg) and average PL wavelength $\left(\lambda_{\text {avg }}\right)$.

could potentially contribute to the QCSE. Firstly, the charge of the ZnS surface itself is a direct function of the $\mathrm{pH}$, becoming more negative as the alkalinity increases [42]. Secondly, changes in the organic passivation layer can alter the surface charge. Recently, the QCSE was demonstrated for QDs coated with a mixed organic capping layer. The QDs, while undergoing ligand exchange, temporarily possessed both amine and thiol ligands, giving rise to an inhomogeneous electric field and a PL redshift [24]. In our case, the pH-dependent dissociation of HDA ligands could lead to a partially (thus inhomogeneous) passivated QD surface. Thirdly, a sulfuration reaction due to acid etching could change the surface charge [14,42]. Fourthly, other chemical species can interact with the QD surface. Carboxylic acids for instance can interact with the surface of thiol-capped CdTe QDs at low pH, resulting in a spectral redshift [20]. Our system contains several carboxylic acid-bearing molecules, including the DHLA and DSPE-PEG ligands as well as buffer acids such as acetate. Finally, the organic ligands (DHLA and DSPE-PEG) contain carboxylic acid groups that will be negatively charged depending on the $\mathrm{pH}$ (e.g. lipoic acid has a pKa of 4.85 [34]).

The $\zeta$-potential can give indirect information on the charge of the organic coating. Figure 6 shows that DHLA-capped and micelle-encapsulated QDs both have a negative $\zeta$-potential over the $\mathrm{pH}$ range $4-9$. The $\zeta$-potential becomes slightly more negative between $\mathrm{pH} 4$ and $\mathrm{pH} 6$ for both coatings, which can be attributed to the deprotonation of the carboxylic acid groups. For micelle-encapsulated QDs, there appears to be no direct correlation between the $\zeta$-potential and the PL wavelength. For DHLA-capped QDs, the data is less conclusive. In the case of micelle-encapsulated QDs, one could assume that the carboxylic acid groups are uniformly spread over the micelle surface. If this is the case, the deprotonation of these groups should not influence the PL spectral position, because for a uniform and spherical charge distribution the net electric field inside is zero. It is therefore likely that the QCSE is due to charges within the micelle.

\section{Effect of the ionic strength on the spectral shift}

In samples with high salinity (i.e. high ionic strength), we observed precipitation of the colloidal QDs after a certain amount of time (hours to days depending on the salt concentration). Salt-induced aggregation occurs because the electric double layer surrounding each particle becomes thinner at high ionic strength, such that the Brownian motion of the particles can temporarily overcome the potential barrier that prevents aggregation. Peculiarly, some samples with high 
ionic strength displayed small shifts of the PL spectrum, similar to the $\mathrm{pH}$-dependent spectral shifts. Because acid or base titrations to adjust the $\mathrm{pH}$ generally also increase the ionic strength of the solution, we investigated the effect of the ionic strength independently of the $\mathrm{pH}$. Figure 7 shows the effect of the ionic strength on the PL wavelength and the hydrodynamic size by varying the $\mathrm{NaCl}$ concentration of a fixed-pH solution. At salt high concentrations ( $\geq 100 \mathrm{mM}$ ), the emission wavelength is red-shifted by $\sim 1 \mathrm{~nm}$. At the same time, the average hydrodynamic particle size increases dramatically, which is attributable to the aggregation of QDs.

We propose two mechanisms by which aggregation could cause a PL redshift. Firstly, particles in aggregates likely find themselves in a non-uniform charge environment. For instance, a particle at the edge of an aggregate borders the solution on one side, but (charged) neighboring particles on the other sides. The resulting electric field could red-shift the $\mathrm{PL}$ emission of that particle. Secondly, the particle separation in aggregates can be much smaller because there is no electric double layer. Consequently, the electric dipoles of neighboring QDs might be coupled resulting in electronic energy transfer. Net energy transfer from smaller to larger QDs then causes a PL redshift. Such energy transfer was shown for deposited QDs making up close-packed QD solids, with 1.1 nm spacing between the dots [43].

\section{Conclusion}

In this study we compared the pH-dependent PL properties of DHLA-capped and micelle-encapsulated QDs. The different organic coatings lead to a different $\mathrm{pH}$-dependence of the PL properties. The $\mathrm{PL}$ quenching in acidic $\mathrm{pH}$ is almost fully reversible for micelle-encapsulated QDs, but nearly irreversible for DHLA-capped QDs. The absence of an irreversible component for micelle-encapsulated QDs could be due to the hydrophobic bilayer protecting the surface against acid etching and oxidation. We postulated that the reversible $\mathrm{pH}$-dependent $\mathrm{PL}$ stems from the surface passivation that is $\mathrm{pH}$ dependent and reversible. Both organic coatings also displayed pH-dependent spectral shifts, which were explained by a combination of irreversible etching and photo-oxidation reactions and reversible polarization of the exciton by local charges (quantum-confined Stark effect). At high ionic strengths, aggregation of QDs causes a spectral redshift, which is due to the quantum-confined Stark effect and/or electronic energy transfer.

Our observations highlight the importance of carefully choosing the organic ligands for colloidal QDs that are to be used in biological or biomedical applications. The influence of $\mathrm{pH}$ and ionic strength on the PL properties should be taken into account and can potentially be exploited in biosensing applications.

\section{Acknowledgement}

This work was supported by the KU Leuven Start grant of C. Bartic and by the KU Leuven BOF concerted research action GOA/14/007. J. Hofkens acknowledges the infrastructure funding of the European Research Council under the European Union's Seventh Framework Programme (FP7/2007-2013)/ ERC Grant Agreement no. 291593 FLUOROCODE) and the Flemish government for the long-term structural funding 'Methusalem' grant METH/08/04 CASAS.

\section{Notes and references}

b Department of Chemistry, KU Leuven, Celestijnenlaan 200F, 3001 Leuven, Belgium. 
* Correspondence (mail): david.debruyne@fys.kuleuven.be

$+\quad$ Electronic Supplementary Information (ESI) available: Transmission electron micrograph of CdSe/ZnS quantum dots; Zetapotential of the water-soluble QDs at neutral pH; PL decay curves of micelle-encapsulated QDs as function of $\mathrm{pH}$; $\mathrm{PH}$-dependence of the average PL lifetime for micelle-encapsulated QDs; Influence of ionic strength on the PL spectrum of DHLA-capped QDs.

[1] Chan W C W, Maxwell D J, Gao X, Bailey R E, Han M and Nie S 2002 Luminescent quantum dots for multiplexed biological detection and imaging. Curr. Opin. Biotechnol. 13 40-6

[2] Michalet X, Pinaud F F, Bentolila L a, Tsay J M, Doose S, Li J J, Sundaresan G, Wu a M, Gambhir S S and Weiss S 2005 Quantum dots for live cells, in vivo imaging, and diagnostics. Science 307 538-44

[3] Walling M a, Novak J a and Shepard J R E 2009 Quantum dots for live cell and in vivo imaging. Int. J. Mol. Sci. 10 441-91

[4] Wang F and Hu S 2009 Electrochemical sensors based on metal and semiconductor nanoparticles Microchim. Acta $1651-22$

[5] Esteve-Turrillas F and Abad-Fuentes A 2012 Applications of quantum dots as probes in immunosensing of smallsized analytes Biosens. Bioelectron. 41 12-29

[6] Jones M, Lo S and Scholes G 2009 Signatures of exciton dynamics and carrier trapping in the time-resolved photoluminescence of colloidal CdSe nanocrystals J. Phys. Chem. C 113 18632-42

[7] Dabbousi B O, Rodriguez-Viejo J, Mikulec F V., Heine J R, Mattoussi H, Ober R, Jensen K F and Bawendi M G 1997 (CdSe)ZnS Core-Shell Quantum Dots: Synthesis and Characterization of a Size Series of Highly Luminescent Nanocrystallites J. Phys. Chem. B 101 9463-75

[8] Reiss P, Protière M and Li L 2009 Core/Shell semiconductor nanocrystals. Small 5 154-68

[9] Murray C and Kagan C 2000 Synthesis and characterization of monodisperse nanocrystals and close-packed nanocrystal assemblies Annu. Rev. Mater. Sci. 30 545-610

[10] Medintz I L, Uyeda H T, Goldman E R and Mattoussi H 2005 Quantum dot bioconjugates for imaging, labelling and sensing. Nat. Mater. 4 435-46

[11] Mattoussi H, Mauro J M, Goldman E R, Anderson G P, Sundar V C, Mikulec F V. and Bawendi M G 2000 SelfAssembly of CdSe-ZnS Quantum Dot Bioconjugates Using an Engineered Recombinant Protein J. Am. Chem. Soc. 122 12142-50

[12] Boldt K, Bruns O T, Gaponik N and Eychmüller A 2006 Comparative examination of the stability of semiconductor quantum dots in various biochemical buffers. J. Phys. Chem. B 110 1959-63

[13] Liu Y-S, Sun Y, Vernier P T, Liang C-H, Chong S Y C and Gundersen M a 2007 pH-sensitive Photoluminescence of $\mathrm{CdSe} / \mathrm{ZnSe} / \mathrm{ZnS}$ Quantum Dots in Human Ovarian Cancer Cells. J. Phys. Chem. C. Nanomater. Interfaces $1112872-$ 8

[14] Durisic N, Godin A G, Walters D, Grütter P, Wiseman P W and Heyes C D 2011 Probing the "dark" fraction of coreshell quantum dots by ensemble and single particle pH-dependent spectroscopy. ACS Nano $59062-73$

[15] Durisic N, Wiseman P W, Grütter P and Heyes C D 2009 A common mechanism underlies the dark fraction formation and fluorescence blinking of quantum dots. ACS Nano 3 1167-75 
[16] Gao X, Chan W W C W and Nie S 2002 Quantum-dot nanocrystals for ultrasensitive biological labeling and multicolor optical encoding. J. Biomed. Opt. 7 532-7

[17] Yu D, Wang Z, Liu Y, Jin L, Cheng Y, Zhou J and Cao S 2007 Quantum dot-based pH probe for quick study of enzyme reaction kinetics Enzyme Microb. Technol. 41 127-32

[18] Spanhel L, Haase M, Weller H and Henglein A 1987 Photochemistry of colloidal semiconductors. 20. Surface modification and stability of strong luminescing CdS particles J. Am. Chem. Soc. 109 5649-55

[19] Kharlampieva E, Kozlovskaya V, Zavgorodnya O, Lilly G D, Kotov N a. and Tsukruk V V. 2010 pH-responsive photoluminescent LbL hydrogels with confined quantum dots Soft Matter 6800

[20] Nanoparticles A C, Zhang H, Zhou Z, Yang B and Gao M 2003 The influence of carboxyl groups on the photoluminescence of mercaptocarboxylic acid-stabilized CdTe nanoparticles J. Phys. Chem. B 107 8-13

[21] Ji X, Palui G, Avellini T, Na H Bin, Yi C, Knappenberger K L and Mattoussi H 2012 On the pH-dependent quenching of quantum dot photoluminescence by redox active dopamine. J. Am. Chem. Soc. 134 6006-17

[22] Coutino-Gonzalez E, Roeffaers M, Bjorn D, De Crememr G, Leyre S, Hanselaer P, Fyen W, Sels B and Hofkens J 2013 Determination and Optimization of the Luminescence External Quantum E ffi ciency of Silver-Clusters Zeolite Composites J. Phys. Chem. C 117 6998-7004

[23] Susumu K, Oh E, Delehanty J B, Blanco-Canosa J B, Johnson B J, Jain V, Hervey W J, Algar W R, Boeneman K, Dawson P E and Medintz I L 2011 Multifunctional compact zwitterionic ligands for preparing robust biocompatible semiconductor quantum dots and gold nanoparticles. J. Am. Chem. Soc. 133 9480-96

[24] Yaacobi-Gross N, Soreni-Harari M, Zimin M, Kababya S, Schmidt A and Tessler N 2011 Molecular control of quantum-dot internal electric field and its application to CdSe-based solar cells. Nat. Mater. 10 974-9

[25] Smith A M, Duan H, Rhyner M N, Ruan G and Nie S 2006 A systematic examination of surface coatings on the optical and chemical properties of semiconductor quantum dots Phys. Chem. Chem. Phys. 83895

[26] Kim S and Bawendi M G 2003 Oligomeric ligands for luminescent and stable nanocrystal quantum dots. J. Am. Chem. Soc. 125 14652-3

[27] Uyeda $\mathrm{H}$ and Medintz I 2005 Synthesis of compact multidentate ligands to prepare stable hydrophilic quantum dot fluorophores J. Am. Chem. Soc. 3870-8

[28] Duan $\mathrm{H}$ and Nie S 2007 Cell-penetrating quantum dots based on multivalent and endosome-disrupting surface coatings. J. Am. Chem. Soc. 129 3333-8

[29] Park K, Deutsch Z, Li J J, Oron D and Weiss S 2012 Single molecule quantum-confined Stark effect measurements of semiconductor nanoparticles at room temperature ACS Nano 6 10013-23

[30] Smith A M and Nie S 2010 Semiconductor nanocrystals: structure, properties, and band gap engineering. Acc. Chem. Res. 43 190-200

[31] Green M 2010 The nature of quantum dot capping ligands J. Mater. Chem. 205797

[32] Kalyuzhny G and Murray R W 2005 Ligand effects on optical properties of CdSe nanocrystals. J. Phys. Chem. B 109 7012-21

[33] Haynes W M 2012 CRC Handbook of Chemistry and Physics (CRC Press) 
[34] Biewenga G, Guido R M M and Bast A 1997 An Overview of Lipoate Chemistry Lipoic Acid in Health and Disease ed J Fuchs, L Packer and G Zimmer (New York: Marcel, Dekker) p 18

[35] Jeong S, Achermann M, Nanda J, Ivanov S, Klimov V I and Hollingsworth J a 2005 Effect of the thiol-thiolate equilibrium on the photophysical properties of aqueous $\mathrm{CdSe} / \mathrm{ZnS}$ nanocrystal quantum dots. J. Am. Chem. Soc. 127 10126-7

[36] Jones M, Nedeljkovic J, Ellingson R J, Nozik A J and Rumbles G 2003 Photoenhancement of Luminescence in Colloidal CdSe Quantum Dot Solutions J. Phys. Chem. B 107 11346-52

[37] Miller D, Chemla D and Damen T 1984 Band-edge electroabsorption in quantum well structures: The quantumconfined Stark effect Phys. Rev. Lett. 2173-6

[38] Park K, Deutsch Z, Li J J, Oron D and Weiss S 2012 Single molecule quantum-confined Stark effect measurements of semiconductor nanoparticles at room temperature. ACS Nano 6 10013-23

[39] Jones M, Lo S S and Scholes G D 2009 Quantitative modeling of the role of surface traps in CdSe/CdS/ZnS nanocrystal photoluminescence decay dynamics. Proc. Natl. Acad. Sci. U. S. A. 106 3011-6

[40] Empedocles S a. 1997 Quantum-Confined Stark Effect in Single CdSe Nanocrystallite Quantum Dots Science (80-. ). $2782114-7$

[41] Braam D, Mölleken A, Prinz G M, Notthoff C, Geller M and Lorke A 2013 Role of the ligand layer for photoluminescence spectral diffusion of CdSe/ZnS nanoparticles Phys. Rev. B 88125302

[42] Wang M, Zhang Q, Hao W and Sun Z-X 2011 Surface stoichiometry of zinc sulfide and its effect on the adsorption behaviors of xanthate. Chem. Cent. J. 573

[43] Kagan C, Murray C, Nirmal M and Bawendi M 1996 Electronic energy transfer in CdSe quantum dot solids. Phys. Rev. Lett. 76 1517-20 